\title{
Promoción de los servicios profesionales farmacéuticos. 'El farmacéutico que necesitas': carpas de salud 2015-2016
}

\author{
Francesc Xavier Moranta Ribas ${ }^{1,2}$, Joaquina Huarte Royo ${ }^{3,4}$ \\ 1. Farmacéutico comunitario. Farmacia Sanz (Palma). 2. Coordinador técnico del proyecto de carpas de servicios profesionales farmacéuticos El \\ farmacéutico que necesitas. 3. Farmacéutica comunitaria. Farmacia Remírez de Ganuza (Pamplona). 4. Adjunta a la coordinación técnica del proyecto \\ de carpas de servicios profesionales farmacéuticos El farmacéutico que necesitas.
}

\section{PALABRAS CLAVE}

Servicios profesionales farmacéuticos, farmacéutico comunitario, farmacia comunitaria, carpas de salud

\section{ABREVIATURAS}

DE: desviación estándar

DIFAC: diabetes y farmacia

comunitaria

EPOC: enfermedad pulmonar obstructiva crónica

FC: farmacéuticos comunitarios

FV: farmacéuticos voluntarios

REFCOM: realidad de la farmacia comunitaria

RUM: revisión del uso de los medicamentos

SEFAC: Sociedad Española de Farmacia Familiar y Comunitaria SNS: Sistema Nacional de Salud SPF: servicio profesional farmacéutico

\section{RESUMEN}

Introducción: La Sociedad Española de Farmacia Familiar y Comunitaria (SEFAC), en colaboración con el laboratorio farmacéutico Ratiopharm, ha llevado a cabo el proyecto de carpas de servicios profesionales farmacéuticos El farmacéutico que necesitas en 2015 y 2016.

Objetivos: Dar a conocer a la sociedad y los pacientes las actividades del farmacéutico comunitario en la prestación de servicios profesionales farmacéuticos (SPF) y valorar su satisfacción con la actividad.

Material y métodos: El proyecto se basa en el ofrecimiento a la población general de actuaciones de SPF adaptados al ámbito de una estructura itinerante, desmontable, tipo carpa. Los servicios ofrecidos fueron: uso adecuado del medicamento, cesación tabáquica, cribado de enfermedad pulmonar obstructiva crónica, consejo nutricional general, cribado de malnutrición en mayores de 65 años, cribado de diabetes, medida de la presión arterial y cálculo del riesgo vascular. Los SPF fueron realizados por farmacéuticos comunitarios (FC) voluntarios locales, previamente formados con protocolos de trabajo diseñados por SEFAC para su actuación en este entorno.

Resultados: El evento se realizó en 15 ciudades diferentes, participaron 655 FC voluntarios, atendiendo a un total de 2.531 usuarios a los que se realizaron 7.290 actuaciones profesionales, con una media de 3 por usuario. La mayoría de los usuarios, encuestados tras recibir los servicios, declararon haber mejorado el conocimiento sobre su estado de salud y lo que desde la farmacia comunitaria se les puede ofrecer.

Conclusiones: Un elevado número de usuarios participantes manifestaron haber incrementado sus conocimientos sobre los SPF y su estado de salud y la práctica totalidad valoró de forma positiva las actividades realizadas.

Este proyecto ha recibido el Premio Fundamed-El Global en Farmacia que reconoce a las mejores iniciativas y proyectos en beneficio de los profesionales farmacéuticos con el objetivo de ayudarles a su mayor integración en el sector sanitario.

Recibido: $15 / 9 / 2017$

Aceptado: $27 / 2 / 2018$

Disponible online: $30-3-2018$
Financiación: el proyecto de carpas de servicios profesionales farmacéuticos El farmacéutico que necesitas cuenta con la colaboración económica del laboratorio Ratiopharm.

Conflicto de intereses: ninguno.

Contribución a la autoría: FXMR participó en el diseño del estudio, el desarrollo de la investigación, el análisis de los resultados, su tratamiento estadistico, la redacción del manuscrito y la revisión del contenido final. JHR en el desarrollo de la investigación, el análisis de los resultados, la redacción del manuscrito y la revisión del contenido final. Cite este artículo como: Moranta FX, Huarte J. Promoción de los servicios profesionales farmacéuticos. 'El farmacéutico que necesitas': carpas de salud 2015-2016. Farmacéuticos Comunitarios. 2018 Mar 30; 10(1):7-17. doi:10.5672/FC.2173-9218.(2018/Vol10).001.03

Correspondencia: Francesc Xavier Moranta Ribas (fmoranta@sefac.org)

ISSN 1885-8619 @SEFAC (Sociedad Española de Farmacia Familiar y Comunitaria). Todos los derechos reservados. 


\section{KEYWORDS}

Professional pharmaceutical services, community pharmacist, community pharmacy, health tents
Promotion of professional pharmaceutical services. "The pharmacist you need": health tents 2015-2016

\section{ABSTRACT}

Introduction: The Spanish Society for Family and Community Pharmacy (SEFAC in Spanish), in collaboration with the Ratiopharm pharmaceutical laboratory, carried out the project of professional pharmaceutical service tents, The Pharmacist You Need, in 2015 and 2016.

Objectives: Raise the awareness of society and patients of the activities of community pharmacists in the provision of professional pharmaceutical services (PPS) and evaluate their satisfaction with the activity.

Materials and Methods: The project is based on offering to the general public PPS actions adapted to the format of a mobile, temporary, tent-type structure. The services offered were: proper use of medicines, smoking cessation, screening for chronic obstructive pulmonary disease, general nutritional advice, screening for malnutrition in people over 65 , screening for diabetes, blood pressure measurement and calculation of vascular risk. The PPS were delivered by volunteer local community pharmacists (CP) who had received prior training on work protocols designed by SEFAC for its action in this setting.

Results: The event was held in 15 different cities, 655 volunteer CPs participated, attending to a total of 2,531 users who received 7,290 professional actions, an average of three per user. Most of the users surveyed after receiving the services said that their awareness of their health status and what a community pharmacy can offer them had improved.

Conclusions: A high number of the users that participated stated that their awareness of PPS and their health status had improved, and practically all of them rated the actions performed positively.

\section{Introducción}

En el año 2013 la Sociedad Española de Farmacia Familiar y Comunitaria (SEFAC), en colaboración con el laboratorio Ratiopharm y el Departamento de Salud Pública de la Facultad de Medicina de la Universidad Complutense de Madrid, promovió el proyecto de investigación REFCOM [1], cuyo objetivo principal era obtener una radiografía lo más precisa posible de la situación profesional y asistencial de las farmacias comunitarias españolas en el que se analizó la opinión de usuarios, asociaciones de pacientes y los propios farmacéuticos comunitarios (FC) sobre diferentes determinantes de la situación real de la farmacia comunitaria en España, mediante la cumplimentación de encuestas específicas.

Los resultados del estudio se presentaron y publicaron en 2014 [1], siendo uno de los más llamativos el bajo nivel de conocimiento y aceptación en general de los servicios profesionales farmacéuticos (SPF) que se pueden ofertar en las farmacias comunitarias. Concretamente el servicio con una menor disposición a utilizar sería el de repaso y detección de los problemas relacionados con los medicamentos (más de un 60\% de la población no estaría dispuesta a utilizarlo en la farmacia). Los demás servicios obtuvieron también porcentajes de aceptación que no llegan al 45\%. Un $44,6 \%$ de la población utilizaría el servicio de deshabituación tabáquica, el 43,5\% el de determinaciones biológicas, y el 41,5\% los relacionados con la educación sanitaria, cribado y prevención de enfermedades [1].

Los servicios profesionales farmacéuticos (SPF) son uno de los asuntos que más debate genera, y han generado en los últimos años en el sector farmacéutico. En SEFAC existe el convencimiento del potencial de los servicios profesionales farmacéuticos asistenciales, por eso es necesario trabajar en ellos no con una perspectiva del futuro de la profesión, sino del presente, desde la formación, la protocolización de los procesos y la evidencia científica. En definitiva, se debe actuar de una forma activa, implantando y desarrollando todos los servicios que sea posible $[2,3]$.
Para que un servicio sea considerado profesional farmacéutico debe conllevar de forma intrínseca un fin asistencial. Según la definición del Foro de Atención Farmacéutica en Farmacia Comunitaria los SPF considerados asistenciales "son aquellas actividades sanitarias prestadas en la farmacia comunitaria por un farmacéutico que emplea sus competencias profesionales para la prevención de la enfermedad y la mejora tanto de la salud de la población como la de los destinatarios de los medicamentos y productos sanitarios”. En definitiva, son servicios alineados con los objetivos del sistema sanitario en el que se enmarcan y deben ayudar a la mejora de la salud del paciente, así como la sostenibilidad del sistema [4].

Por otra parte, para que los SPF ofrezcan garantías de calidad y seguridad deben estar protocolizados y consensuados con las sociedades científicas de otros profesionales sanitarios que puedan intervenir en el proceso de atención al paciente [5]. El profesional que los realice debe estar capacitado para ello con el fin de 
que resulten eficaces, pueda medirse su eficiencia, ser incorporados en la cartera de prestaciones del Sistema Nacional de Salud (SNS) y lograr su retribución por este [5].

Los resultados obtenidos del estudio REFCOM revelan que se puede mejorar el conocimiento y la aceptación por parte los pacientes sobre los servicios ofrecidos en la farmacia, y la necesidad de comprobar la viabilidad de los procedimientos diseñados para su prestación. Por ello, partiendo de la experiencia adquirida en la organización y realización de carpas de salud, se decidió crear el proyecto de promoción de los servicios profesionales farmacéuticos El farmacéutico que necesitas. En el presente estudio se presentan los resultados globales de las acciones de promoción realizadas dentro de este proyecto en los años 2015 y 2016.

\section{Objetivos}

Dar a conocer los servicios profesionales asistenciales que pueden prestarse en las farmacias comunitarias por farmacéuticos comunitarios a la población en general.

Ofertar y proveer estos servicios a posibles usuarios, valorando su opinión sobre los servicios recibidos $\mathrm{y}$ conocer si estos serían bien recibidos si se los ofrecieran en su farmacia comunitaria de manera habitual.

\section{Materiales y método Diseño}

Estudio observacional transversal de las acciones realizadas durante los años 2015 y 2016 en quince ciudades españolas.

\section{Selección de los servicios}

Se seleccionaron SPF según las características del ámbito en el que se iba a realizar el proyecto, así como el grado de capacitación que se iba obteniendo entre los farmacéuticos comunitarios mediante la formación impartida en el Campus SEFAC. Todos ellos se basaron en proyectos liderados por SEFAC en los que se llevaba años trabajando, como CESAR [6,7] (servicio de cesación tabáquica), ImpacHta [8,9] (hipertensión arterial y riesgo vascular), Revisa [10-12] (Revisión del Uso de los Medicamentos) (RUM), DIFAC [13,14] (cribado y seguimiento de la diabetes), en colaboración con otras sociedades científicas y que combinan la formación continuada teórica con la práctica y el registro de casos clínicos. En otros casos, se basaron en estudios de investigación de los Grupos de trabajo de SEFAC o de sus delegaciones (D'Nut [15], Farbalepoc [16]).

\section{Formación de los voluntarios}

Para cada carpa se eligieron dos coordinadores generales a los que se formó para asumir la responsabilidad del desarrollo del proyecto en las carpas. Estos designaron y formaron a los coordinadores de cada área de salud que, a su vez, formaron a todos los farmacéuticos voluntarios participantes.

\section{Protocolo y circuitos}

La dirección técnica del proyecto en colaboración con los grupos de trabajo de SEFAC, diseñó el protocolo del estudio así como los procedimientos que había que seguir. Se pretendía lograr la extrapolación y la reproductibilidad de las actuaciones y registros en todas las carpas.

Zona de captación/bienvenida: al paciente se le acogía en la zona de bienvenida de la carpa. Allí se le informaba sobre el estudio y los servicios profesionales que podía recibir en función de su edad, su estado de salud, las patologías diagnosticadas y los tratamientos prescritos. En esa misma zona, se le entregaba al paciente la hoja de registro donde el farmacéutico de acogida anotaba los servicios que iba a recibir el paciente, sus características demográficas, etcétera.

Una vez el usuario accedía a recibir cada uno de los servicios, se comprobaban según protocolo los requisitos de inclusión al mismo y se le realizaba o no. Al proveer el servicio, el farmacéutico comunitario podía detectar situaciones en las que era necesario derivar el paciente atendido al médico. En estos casos, el farmacéutico cumplimentaba una hoja de informe de derivación al médico y se le indicaba de viva voz que era necesario que acudiera a su médico de cabecera. Además, al finalizar la provisión de los servicios, se entregaba en este caso a todos los pacientes participantes un material escrito de consejos y recomendaciones de hábitos saludables. Finalmente, y antes de abandonar la carpa, se trataba de realizar una encuesta de satisfacción a todos los pacientes que habian participado.

\section{Materiales y medios humanos \\ La carpa: es la estructura física,} itinerante y desmontable, en el interior de la cual se realizó el proyecto (figura 1). Las carpas fueron levantadas en plazas céntricas de las diferentes ciudades elegidas, habiendo una sola zona de entrada y salida, donde se

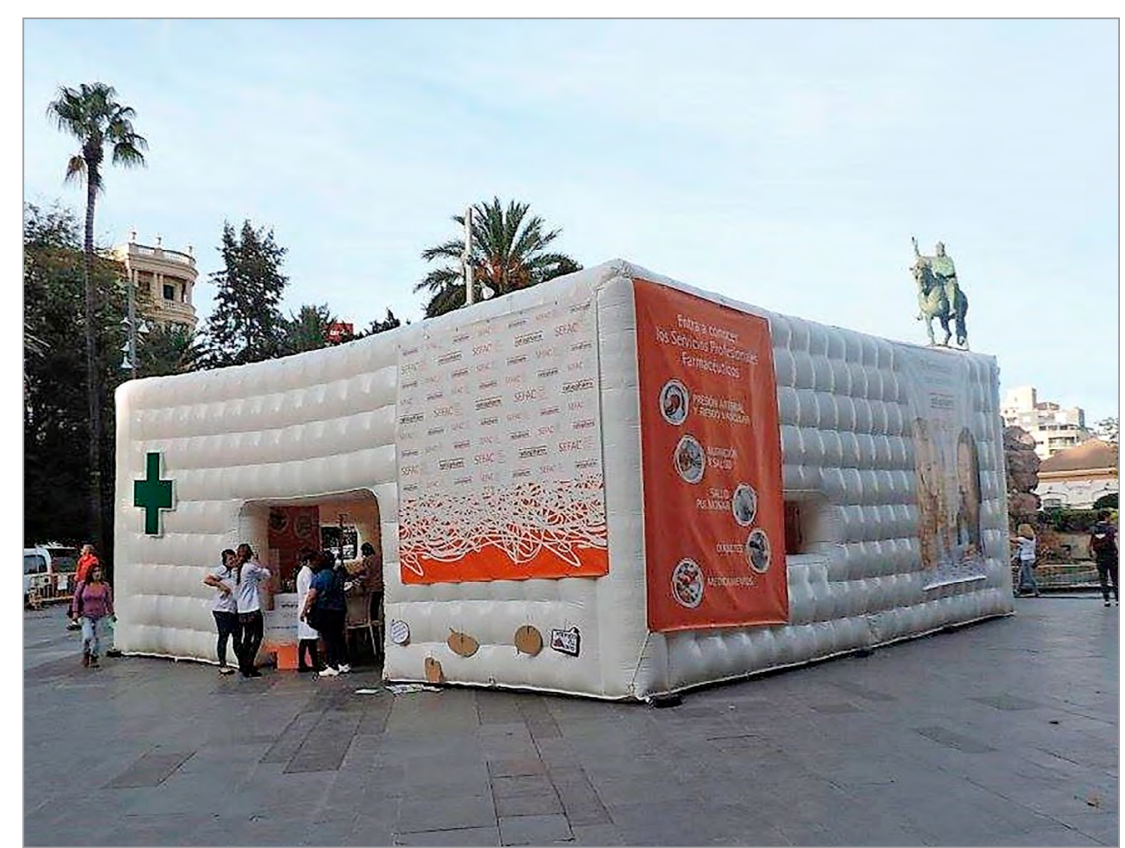

Figura 1 Estructura desmontable tipo carpa 
sitúa la zona de captación/bienvenida y en su interior se dispusieron las cinco áreas temáticas. El horario de atención a los pacientes quedaba comprendido entre las 10:00 h de la mañana y las 18:00 h de la tarde, aproximadamente, de forma ininterrumpida, registrándose los datos correspondientes durante el mismo horario.

Hoja de registro: era el documento codificado con numeración correlativa que se entregaba al paciente en la zona de bienvenida. En dicha hoja se registraba, entre otros, la fecha y el nombre de la ciudad, se indicaban los servicios que había que proveer y se anotaban todas las variables necesarias para el estudio. Al finalizar el circuito el usuario entregaba la hoja de registro en el control de salida y se quedaba con una copia (anexo 1).

Encuesta de satisfacción: al finalizar el circuito se invitaba al usuario a cumplimentar una encuesta de satisfacción sobre los servicios recibidos. Se utilizaba un cuestionario estructurado de seis preguntas, en su mayoría cerradas dicotómicas o de elección múltiple (anexo 2).

Material de soporte: en cada servicio se entregaban hojas informativas con consejos y recomendaciones sobre determinadas patologías y hábitos saludables editadas por SEFAC.

Hojas de derivación: eran las hojas específicas para derivar el pa- ciente a su farmacia comunitaria habitual o al médico de cabecera si era necesario y según los resultados obtenidos en la provisión del servicio (anexo 3).

Farmacéuticos voluntarios (FV): todos los servicios fueron ofrecidos $\mathrm{y}$ realizados por $\mathrm{FV}$, que recibieron formación previa sobre el funcionamiento general de la carpa y especifica sobre los protocolos que había que cumplir en el servicio concreto al que fueron asignados.

Protocolos y documentación: como se ha comentado anteriormente se trataba de todos los documentos (algoritmos, protocolos, documentación del servicio, hojas de derivación y registro, esquemas, instrucciones de uso de los diferentes aparatos, presentaciones para la formación, etc.) y las hojas de información que fueron elaborados por los diferentes grupos de trabajo de SEFAC, y la dirección técnica de este proyecto. Se utilizó como base el material, de los proyectos y estudios SEFAC en los que se basan dichos servicios (CESAR, EPOCA, ImpacHta, DIFAC, Farbalepoc, D'Nut) adaptados al ámbito de una carpa de salud y acondicionados al formato de episodio de SPF.

Población de estudio: la carpa de SPF es un proyecto abierto a la población general. Los servicios de medida de la presión arterial, la frecuencia cardiaca y el consejo nutricional se ofrecían a todos los usuarios que se acercaban a la carpa. En cambio, para ofrecer y prestar los otros servicios, era necesario cumplir determinadas características de inclusión:

- El servicio de uso adecuado del medicamento estaba orientado a pacientes que tomasen algún medicamento.

- En cálculo del riesgo cardiovascular era necesario no estar clasificado como de riesgo alto por determinadas circunstancias o factores, $\mathrm{y}$ pertenecer a la franja de edad adecuada para cada sistema de cálculo: Regicor o Score.

- Para cesación tabáquica había que ser un fumador activo.

- Para cribado de EPOC, tener entre 40 y 80 años, ser fumador, exfumador o fumador pasivo y presentar síntomas o signos crónicos como tos, disnea, y/o expectoración.

- En detección de desnutrición, ser mayor de 65 años.

- Para cribado de diabetes, tener 18 o más años y no estar diagnosticado de diabetes y/o estar en tratamiento con hipoglucemiantes o insulinas.

\section{Resultados}

Las carpas se realizaron desde junio de 2015 a diciembre de 2016 en 15 ciudades españolas (figura 2).

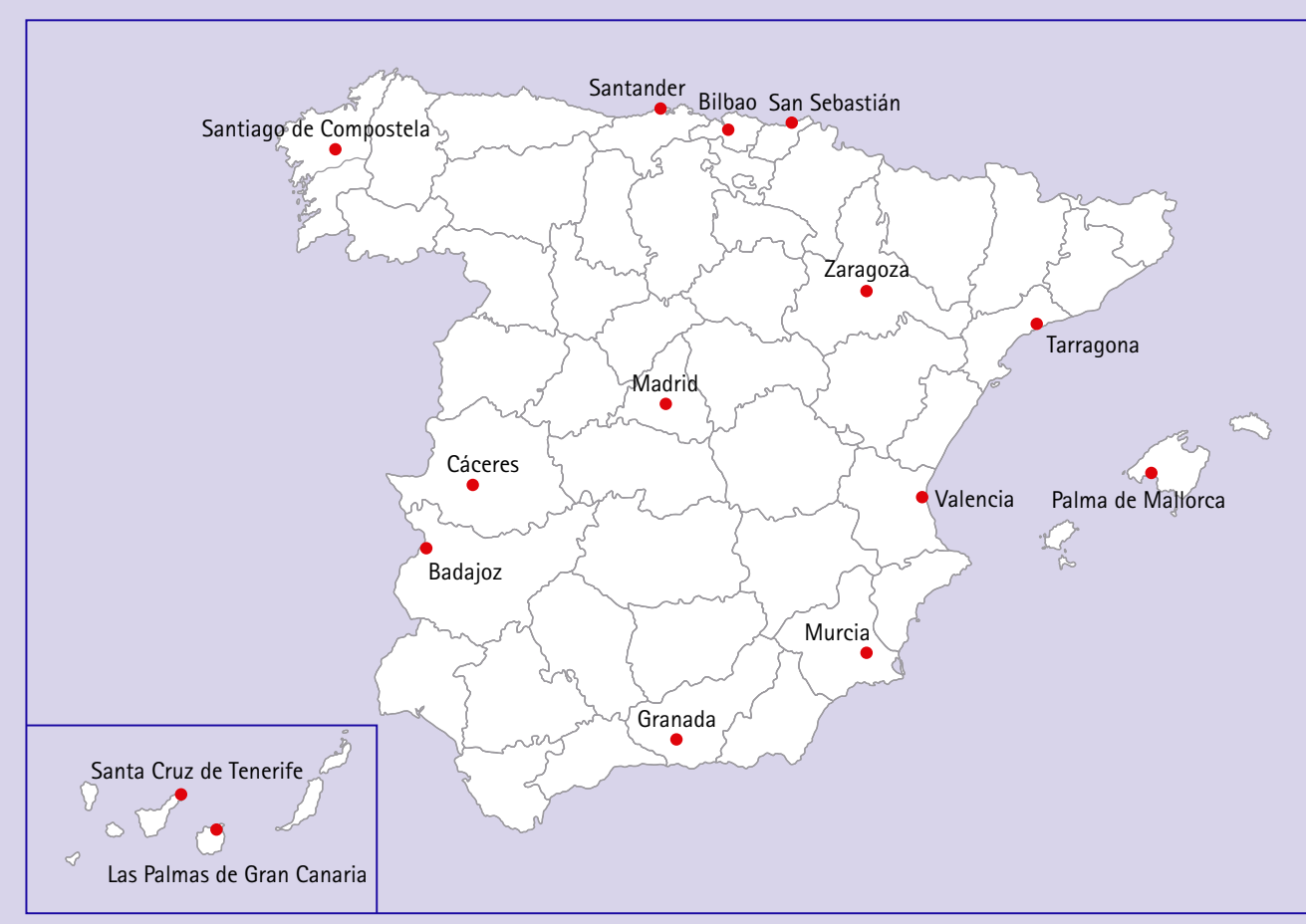

Carpas 2015

Las Palmas de Gran Canaria San Sebastián

Zaragoza

Palma de Mallorca

Tarragona

Valencia

Carpas 2016

Madrid

Badajoz

Santander

Santiago de Compostela

Bilbao

Granada

Cáceres

Santa Cruz de Tenerife

Murcia

Figura 2 Mapa de la distribución geográfica de las carpas de salud 2015-2016 
Tabla 1 Número de farmacéuticos voluntarios por localización del evento

\begin{tabular}{|l|c|}
\hline Localidad & $\begin{array}{c}\text { Número de FC } \\
\text { voluntarios }\end{array}$ \\
\hline Granada & 72 \\
\hline Las Palmas de Gran Canaria & 56 \\
\hline Santa Cruz de Tenerife & 52 \\
\hline Badajoz & 49 \\
\hline Murcia & 49 \\
\hline Madrid & 47 \\
\hline Valencia & 45 \\
\hline Santiago de Compostela & 44 \\
\hline Palma de Mallorca & 42 \\
\hline Zaragoza & 37 \\
\hline Bilbao & 36 \\
\hline Santander & 35 \\
\hline Tarragona & 33 \\
\hline Cáceres & 655 \\
\hline San Sebastián & \\
\hline TOTAL & 32 \\
\hline
\end{tabular}

Participaron 655 farmacéuticos comunitarios voluntarios, con una media de 44 por ciudad, con un máximo de 72 en Granada y un mínimo de 26 en San Sebastián (tabla1).

El total de usuarios registrados fue de 2.531 con una media de 169 por localización, con un mínimo de 100 en Tarragona y un máximo de 275 en Madrid. La media de edad fue de 56,7 (DE=-18,5) años, siendo $982(38,8 \%)$ mayores de 65 años. El $56,1 \%$ de los usuarios participantes fueron mujeres.

Se realizaron un total de 7.290 SPF, con una media de 2,9 por usuario. El servicio de medida de la presión arterial y frecuencia cardiaca fue el que más actuaciones registró, 1.914 , lo que representa un 75,6\% del total de usuarios de las carpas.

La distribución de los servicios realizados a los 2.531 usuarios que se registraron se muestra en la tabla 2.

Con respecto a las 2.160 encuestas de satisfacción recogidas, del 85,4\% de los usuarios totales que recibieron SPF en las carpas, los resultados más destacados son:

- El 61,6\% desconocía con anterioridad los servicios que le habían prestado en las carpas.
Tabla 2 Distribución de los servicios realizados

\begin{tabular}{|l|c|}
\hline SPF & $\begin{array}{c}\text { Actuaciones } \\
\text { Medida de la presión arterial y la } \\
\text { frecuencia cardiaca }\end{array}$ \\
\hline Consejo nutricional & $1.914(26,3)(75,6)$ \\
\hline Cribado de diabetes & $1.651(22,6)(65,2)$ \\
\hline Uso adecuado del medicamento & $1.217(16,7)(48,1)$ \\
\hline Cálculo del riesgo cardiovascular & $1.070(14,7)(42,3)$ \\
\hline Cribado de EPOC & $541(7,4)(21,4)$ \\
\hline Cesación tabáquica & $448(6,1)(17,7)$ \\
\hline Cribado de desnutrición & $313(4,3)(12,4)$ \\
\hline TOTAL & $136(1,9)(5,4)$ \\
\hline
\end{tabular}

- Un 60,5\% declaraba no recibir servicios en las farmacias a las que acudían habitualmente

- El 39,5\% restante que sí declaraban recibirlos indicaban que los servicios que recibian eran los siguientes: medida de la presión arterial, un 22,5\%; determinación del colesterol, un 4,4\%; medida del peso y del IMC, un 4,0\%; determinación de la glucemia, un 4,0\%; consejos sobre el uso adecuado de la medicación, un 1,8\%, y cesación tabáquica, un $0,8 \%$.

Después de realizar el recorrido por la carpa recibiendo los servicios asignados el 92,3\% de los usuarios declaró haber mejorado sus conocimientos relativos a los servicios ofrecidos y el 88,3\% de ellos declaró en consecuencia directa conocer mejor su estado de salud. El 76,8\% estaría dispuesto a desembolsar una cantidad económica para obtener los servicios, un $0,6 \%$ dependería de cada caso concreto y un 22,6\% no estaría dispuesto en ningún caso.

En cuanto a la calidad de los servicios que se les habian prestado, la práctica totalidad de los usuarios los valoró como buenos o muy buenos en general (figura 3).

\section{Discusión}

En este proyecto se han presentado diferentes limitaciones que se comentarán al hilo de esta discusión y que no nos permiten evaluar de manera absoluta el cumplimiento de alguno de los objetivos propuestos.

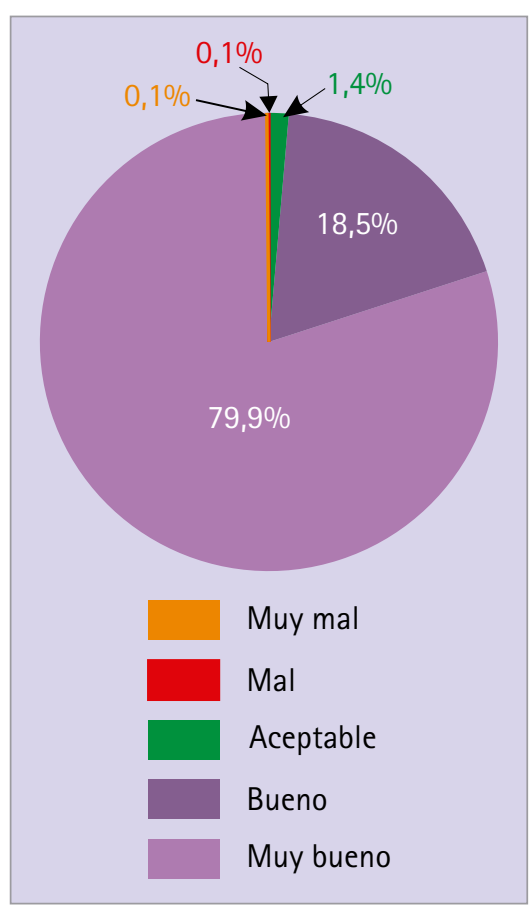

Figura 3 Valoración general de los servicios recibidos por los pacientes/usuarios

En otros casos, sin embargo, sí que se han cumplido objetivos concretos.

En cuanto al primer objetivo del proyecto, que era dar a conocer la prestación de determinados SPF en las farmacias comunitarias para aumentar su grado de conocimiento por la población general, de forma objetiva y de acuerdo al método científico se ha de asumir que sin repetir exactamente el mismo método utilizado en el proyecto REFCOM [1] es inviable comprobarlo.

El proyecto de "carpas de servicios profesionales farmacéuticos" está difundiendo y promocionando la 


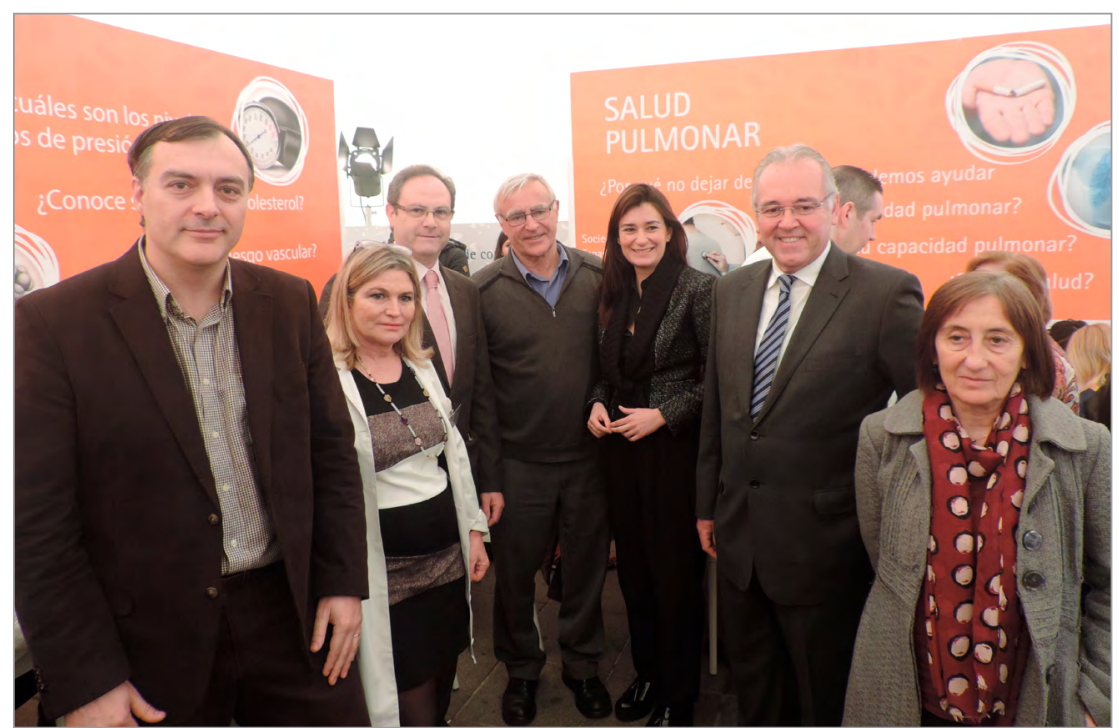

El alcalde de Valencia, Joan Ribó, y la consejera de Sanidad Universal y Salud Pública de la Comunitat Valenciana, Carmen Montón (en el centro), durante su visita a la carpa celebrada en Valencia

implantación y el ejercicio de estos servicios en las farmacias comunitarias españolas y será difícil discriminar qué parte corresponde en esa difusión a este proyecto concreto. En cualquier caso, está claro que la repetición del estudio REFCOM en unos años sería muy importante para poder medir el nivel de penetración de estos servicios en la sociedad en general y en el sistema sanitario español en particular. También es cierto que contemplando los números del proyecto de una forma subjetiva podríamos estar de acuerdo por su magnitud (655 FV, 2.531 usuarios participantes y 7.290 SPF) y por la amplitud de la distribución geográfica de las carpas de SPF en que el impacto del proyecto, aunque difícilmente medible, es más que evidente, también por el amplio respaldo que esta iniciativa ha tenido institucionalmente, por la presencia en las carpas de numerosas autoridades (representantes de ayuntamientos, consejerías, colegios de

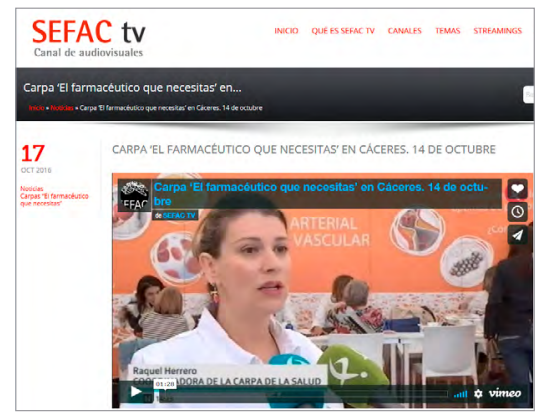

'El farmacéutico que necesitas' (Cáceres, (3) 14 de octubre de 2017) cendencia del proyecto en sí radicaba en la calidad de los servicios oferta- dos, que creemos se ha visto reflejada en los registros obtenidos.

Por otro lado, creemos que ha tenido una repercusión efectiva en medios de comunicación y redes sociales cuyas dimensiones no podemos cuantificar, pero que se ha visto reflejada en las reseñas de medios escritos y audiovisuales que informaban de la realización de la actividad.

En cuanto al segundo objetivo, destaca la alta demanda de los de medida de la presión arterial y frecuencia cardiaca, consejo nutricional, y cribado de diabetes. No obstante debido a evidentes sesgos del proyecto es necesario hacer una serie de apreciaciones sobre algunos de ellos, pues se han encontrado algunas limitaciones que pueden condicionar los resultados obtenidos. La más importante ha sido su realización casi exclusivamente (excepto Bilbao) en días laborables y mayoritariamente de 10:00 a 18:00 h, por lo que no se ha podido caracterizar una muestra de estudio representativa para cada servicio, ya que al ser un día y un horario eminentemente laboral y/o escolar se ha recogido una muestra general de edad más avanzada de la esperada (56,7 años). Este hecho ha podido influir en los resultados generales del proyecto. También ha podido influir un hecho que se repitió en todas las carpas, las acumulaciones puntuales de usuarios en determinadas horas y en determinados servicios. En consecuencia, por cuestiones de disponibilidad horaria, no todos ellos pudieron recibir todos los servicios a los que se les había asignado. Este hecho puede haber influido en la evaluación de la demanda de determinados servicios, siendo el área de salud pulmonar la más afectada, si atendemos a la prevalencia del tabaquismo en España (23\% de fumadores mayores de 16 años) [17],

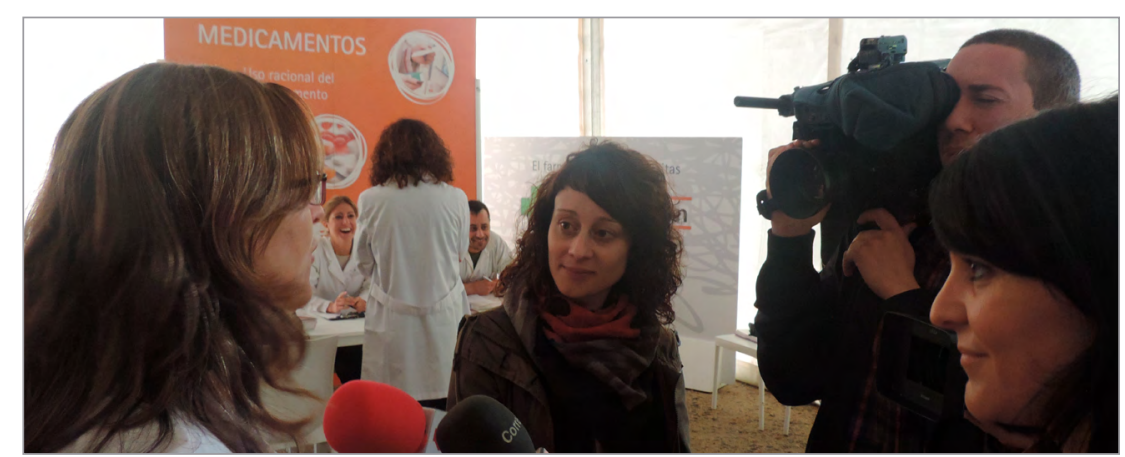

Visita de los medios de comunicación gallegos a la carpa celebrada en Santiago de Compostela 
con números absolutos y porcentajes relativos menores de lo esperado (12,4\% de los usuarios). Aunque también podría atribuirse esta baja demanda a la propia idiosincrasia psicológica del fumador, y a la negación del problema de salud o patología. En esta misma área en el servicio de cribado de EPOC, no se pudo realizar el servicio completo a $23(5,1 \%)$ de los pacientes a los que se debía de realizar la prueba de expiración forzada debido a posibles incidencias relacionadas con el emplazamiento físico del servicio, y a un entorno inadecuado. Aunque es un porcentaje reducido podría implicar un cierto menoscabo de su viabilidad a la hora de utilizar este servicio en el ámbito de una carpa, no así en el de la farmacia comunitaria como se ha demostrado en diferentes estudios [18]. Otro servicio con demanda menor de lo esperada es el de uso adecuado del medicamento, con apenas un 42\% de casos registrados sobre el total de participantes en el proyecto. Más aún si cabe, si atendemos a que es uno de los servicios que tenía una población de estudio teórica más amplia (cualquier persona usuaria de medicamentos). Estos resultados están en consonancia con los registrados en el estudio REFCOM [1] donde los servicios sobre revisión de la medicación y uso adecuado del medicamento eran apreciados por menos del $40 \%$ de los encuestados. Creemos que este dato es muy preocupante y constata que los posibles usuarios de la farmacia comunitaria no ven la necesidad, ni conocen los beneficios de los SPF más directamente relacionados con los medicamentos y con la razón de ser del farmacéutico. El servicio menos demandado de los ocho ofertados ha sido el de detección de desnutrición en mayores de 65 años. Considerando que la población teórica de estudio a la que se asignó el servicio era de 982 posibles usuarios (mayores de 65 años participantes en el proyecto), solamente se prestó este servicio a 136 usuarios, lo que representa un $13,8 \%$ sobre la totalidad de mayores de 65 años que participaron en el proyecto, aunque estudios como el D'Nut constaten que la desnutrición en este colectivo presenta una prevalencia mucho más elevada de lo que cree la sociedad y los mismos afectados con prevalencia de malnutrición de $0,8 \%$ y un riesgo de malnutrición de 12,2\% en mayores de 65 años [15].

En cuanto a la valoración y disposición de los usuarios con los servicios recibidos, casi un $40 \%$ de los pacientes declaro recibir algún servicio en su farmacia habitual. Sin embargo, al indicar cuál era el servicio que recibian, la mayoría realmente hizo referencia a mediciones, no a servicios asistenciales profesionales farmacéuticos propiamente dichos. Con lo cual se constata lo que ya se reflejaba en el estudio REFCOM [1], el bajo nivel de conocimiento de la población general sobre los servicios que pueden ofrecerse en la farmacia comunitaria pero, además, la falta de información para poder diferenciar entre mediciones simples de diferentes parámetros y lo que consideramos propiamente como un SFP. Por tanto, creemos que el porcentaje real de usuarios de las carpas que realmente reciben SPF es muchísimo menor de lo expresado por ellos mismos. En la vertiente positiva se constata la alta aceptación y valoración de los SPF, ya que prácticamente la totalidad de los pacientes valoran como buenos o muy buenos los servicios recibidos, y que tres cuartas partes de ellos estarían dispuestos a pagar una cantidad económica para obtenerlos, aunque no se establecieron cuantías económicas para ninguno de ellos.

\section{Agradecimientos}

Este es un proyecto coral, no de unos pocos iluminados, sino de toda una sociedad científica y del colectivo de farmacéuticos comunitarios, en el que han participado más de 1.000 farmacéuticos en diferentes niveles. Nuestro agradecimiento a todos y cada uno de ellos, en especial a los 30 coordinadores generales de carpa, a los más de 130 coordinadores de área o servicio, a los más de 650 farmacéuticos voluntarios, y a todos los responsables y miembros de los grupos de trabajo de SEFAC que han intervenido en la definición de protocolos, procedimientos de trabajo y algoritmos. A todos y cada uno de ellos nuestro agradecimiento por participar en un proyecto del que los farmacéuticos comunitarios nos podemos sentir especialmente orgullosos.

Nuestro reconocimiento también a los Colegios Oficiales de Farmacéuticos de cada provincia donde se realizó el proyecto, sin su inestimable colaboración y ayuda en la promoción del acto y formación de los farmacéuticos voluntarios, hubiera sido imposible su realización.

Por último una mención especial para el Staff de SEFAC asignado al proyecto, Alicia Martínez y Mario Vaillo, que se han recorrido España entera durante el transcurso del proyecto, con una dedicación que va más allá de su deber profesional. 
Anexo 1. Hoja de registro de los servicios recibidos

\section{HOJA DE REGISTRO CARPA SEFAC}

$\begin{array}{llll} & & & \\ \text { - CIUDAD: } & \text { FECHA: } & \text { - No Registro: } \\ \text { - Sexo: } & \text { [ ] Mujer } & \text { [ ] Hombre } & \text { - Edad: }\end{array}$

1.1 Medida de la PA
\begin{tabular}{|l|l|l|l|l|}
\hline $1^{\mathrm{a}}$ & $2^{\mathrm{a}}$ & Media $^{*}$ & $3^{\mathrm{a}}$ & Media $^{*}$ \\
\hline PAD_PAS_FC__ & PAD_PAS_FC__ & PAD_PAS_FC_ & PAD_PAS_FC_ & PAD_PAS_FC__ \\
\hline
\end{tabular}

1.2 Cálculo del RV mayores de 40 años

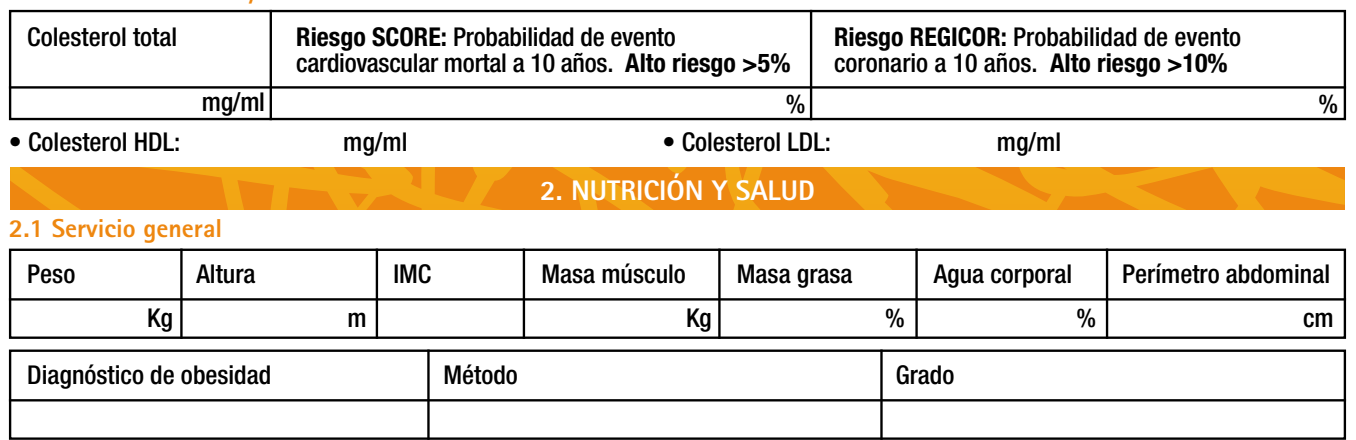

2.2 Detección de desnutrición mayores de 65 años

\begin{tabular}{|l|l|}
\hline Resultado test MNA & Riesgo desnutrición \\
\hline & \\
\hline
\end{tabular}

\begin{tabular}{|r|l|l|}
\hline \multicolumn{3}{|c|}{ 3. GLUCEMIA Y DIABETES } \\
\hline Glucemia (basal o postpandrial) & HBA1C & Resultado test FINDRISC \\
\hline $\mathrm{mg} / \mathrm{ml}$ & & \\
\hline
\end{tabular}

4. SALUD PULMONAR

4.1. Cesación tabáquica en fumadores

\begin{tabular}{|r|r|r|l|l|}
\hline Edad pulmonar & Cooximetría CO & Cooximetría CO-Hb & Test de Fageström & Test de Richmond \\
\hline años & $\mathrm{ppm}$ & $\%$ & & \\
\hline
\end{tabular}

4.2. Cribado de EPOC en fumadores, exfumadores y fumadores pasivos

\begin{tabular}{|c|c|c|c|c|c|c|c|c|c|c|c|}
\hline Test COPD-PS & & & \multicolumn{2}{|c|}{ FEV1/FEV6 } & & & & \multicolumn{2}{|c|}{ Edad pulmonar } & \multicolumn{2}{|c|}{ años } \\
\hline \multicolumn{12}{|c|}{ 5. USO ADECUADO DEL MEDICAMENTO } \\
\hline \multicolumn{3}{|c|}{ SERVICIO / Entrevista Medicamentos genéricos } & \multicolumn{8}{|c|}{ INSTRUCCIONES AL PACIENTE / Entrevista Uso adecuado del medicamento } & \\
\hline $\begin{array}{ll}01 . & \text { V [ } \\
02 . & \text { V }[ \\
03 . & \text { V [ } \\
04 . & \text { V [ } \\
05 . & \text { V [ }\end{array}$ & $\begin{array}{l}\mathbf{F}[] \\
\mathrm{F}[\mathrm{]} \\
\mathrm{F}[\mathrm{]} \\
\mathrm{F}[\mathrm{]} \\
\mathrm{F}[\mathrm{]}\end{array}$ & $\begin{array}{l}\text { ns [ ] } \\
\text { ns [ ] } \\
\text { ns [ ] } \\
\text { ns [ ] } \\
\text { ns [ ] }\end{array}$ & $\begin{array}{l}01 . \\
02 . \\
03 . \\
04 . \\
05 .\end{array}$ & $\begin{array}{l}\text { Si [ ] } \\
\text { Si [ ] } \\
\text { Si [ ] } \\
\text { Si [ ] } \\
\text { Si [ ] }\end{array}$ & $\begin{array}{l}\text { No [ ] } \\
\text { No [ ] } \\
\text { No [ ] } \\
\text { No [ ] } \\
\text { No [ ] }\end{array}$ & $\begin{array}{l}\text { ns [ ] } \\
\text { ns [ ] } \\
\text { ns [ ] } \\
\text { ns [ ] } \\
\text { ns [ ] }\end{array}$ & $\begin{array}{l}06 . \\
07 . \\
08 . \\
09 . \\
10 .\end{array}$ & $\begin{array}{l}\text { Si[ ] ] } \\
\text { Si[ ] } \\
\text { Si [ ] } \\
\text { Si[ ] } \\
\text { Si[ ] }\end{array}$ & $\begin{array}{l}\text { No [ } \\
\text { No [ } \\
\text { No [ } \\
\text { No [ } \\
\text { No [ }\end{array}$ & $\begin{array}{l}\text { ns [ ] } \\
\text { ns [ ] } \\
\text { ns [ ] } \\
\text { ns [ ] } \\
\text { ns [ ] }\end{array}$ & 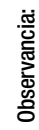 \\
\hline
\end{tabular}

\begin{tabular}{|c|c|}
\hline Servicio & Motivo derivación \\
\hline & \\
\hline & \\
\hline & \\
\hline & \\
\hline
\end{tabular}




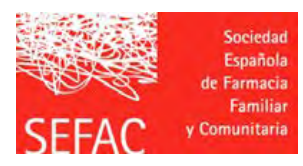

\section{Encuesta de satisfacción - Carpas SPF}

Con el objetivo de conocer la calidad del servicio que hemos ofrecido, así como mejorar en nuestro trabajo del día a día, agradeceríamos que nos contestase a una serie de preguntas.

¿Conocía usted los servicios que se pueden ofrecer desde las farmacias comunitarias?

$\square$ No $\square$ Sí

¿Recibe usted alguno de estos servicios profesionales en su farmacia habitual?

$\square$ No $\quad \square$ Sí $\quad$ ¿Cuál?

¿Ha mejorado su conocimiento relativo a los servicios ofrecidos después de este recorrido?

$\square$ No $\quad \square$ Sí

¿Estaría dispuesto a desembolsar una cantidad económica para obtener este servicio?

$\square$ No $\square$ Sí

¿Conoce usted mejor su estado de salud después de realizar el recorrido?

$\square$ No $\quad \square$ Sí

Valore el servicio ofrecido en esta carpa en la sección de:

- Uso adecuado del medicamento:

$\square$ Muy bueno $\square$ Bueno $\quad \square$ Aceptable $\square$ Malo $\quad \square$ Muy malo

- Salud pulmonar:

$\square$ Muy bueno $\square$ Bueno $\quad \square$ Aceptable $\square$ Malo $\quad \square$ Muy malo

- Salud cardiovascular:
$\square$ Muy bueno
$\square$ Bueno
$\square$ Aceptable
$\square$ Malo
$\square$ Muy malo

- Diabetes:

$\square$ Muy bueno $\quad \square$ Bueno $\quad \square$ Aceptable $\quad \square$ Malo $\quad \square$ Muy malo

- Nutrición y salud:

$\square$ Muy bueno

$\square$ Bueno $\square$ Aceptable $\square$ Malo $\quad \square$ Muy malo 
Anexo 3. Ejemplo de folleto de recomendaciones SEFAC a la población

Recomendaciones de la Sociedad Española de Farmacia Familiar y Comunitaria a la población.

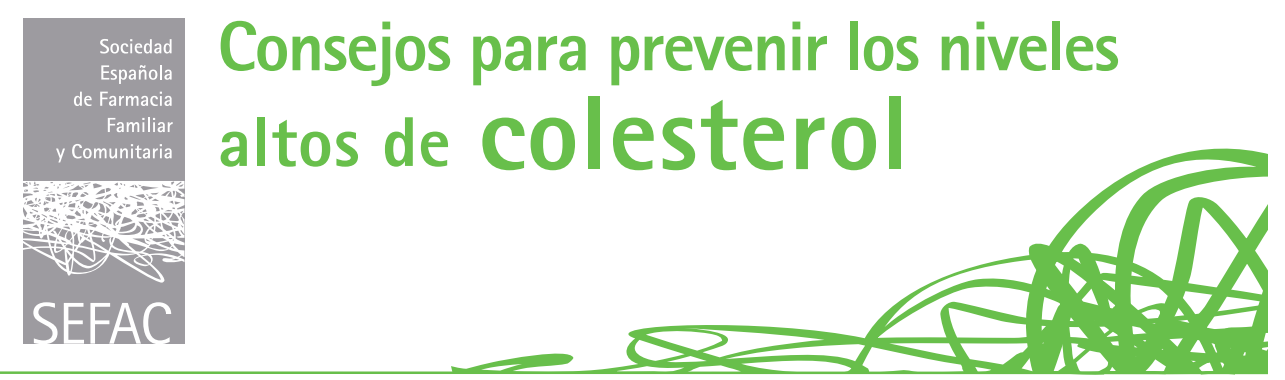

\section{Proteja su corazón}

El colesterol es una sustancia grasa natural que está presente en todas las células del cuerpo y se necesita para formar tejidos celulares, producir ciertas hormonas y facilitar la digestión de las grasas. A pesar de que es necesario para nuestro organismo, demasiado colesterol en la sangre aumenta el riesgo de sufrir un ataque al corazón o un infarto cerebral. Las enfermedades cardiovasculares representan la primera causa de mortalidad en el mundo y la segunda causa de años potenciales de vida perdida y de mortalidad prematura después del cáncer.

Los niveles de colesterol tienden a crecer con la edad. El aumento de colesterol no suele tener signos ni síntomas, pero puede detectarse con un simple

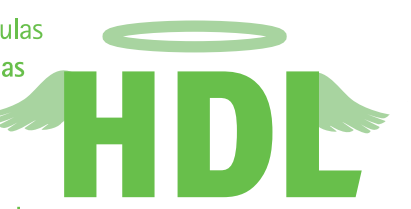
análisis de sangre. Usted tendrá más probabilidades de tener un nivel de colesterol alto si tiene antecedentes familiares, sobrepeso o abusa de las comidas grasas.

Una dieta adecuada, hacer ejercicio físico y dejar de fumar son las medidas más efectivas para bajar el colesterol y los triglicéridos. Además, estos cambios en el estilo de vida son para siempre y deberá seguirlos aunque tome medicamentos para controlar el colesterol.

\section{Recomendaciones}

SIGA UNA DIETA VARIADA, con abundancia de cereales, verduras $y$ frutas.

REDUZCA el sobrepeso. Se ha demostrado que las personas obesas tienen mayor riesgo de padecer infartos y obstrucciones arteriales que la población general no obesa.

DISMINUYA EL CONSUMO DE HUEVOS (máximo 2 ó 3 por semana), leche entera y derivados (helados, nata, mantequilla, yogures enteros, quesos grasos...).

AUUSTE EL CONSUMO de carnes rojas a dos días por semana y el de pollo, pavo sin piel o conejo a dos o tres dias por semana, y nunca en cantidades superiores a los 200 gramos. INTRODUZCA EN SU DIETA los pescados blancos y en especial los azules (sardina, trucha, atún, caballa, salmón...). EVITE EN LO POSIBLE los fritos y guisos, y cocine preferiblemente a la plancha o a la brasa, retirando la grasa visible de la carne antes de cocinarla.

Utilice LA SAL con moderación. El exceso de sodio es muy dañino para nuestro corazón y nuestras arterias.

EVTEEL CONSUMO de productos de bolleria, fritos y precocinados, ricos en ácidos grasos saturados que perjudican su salud. CONTROLE EL CONSUMO de alcohol. Es aceptable en los adultos hasta dos vasos de vino al día, pero el alcohol en general está desaconsejado en pacientes con sobrepeso, mujeres embarazadas y pacientes con hipertrigliceridemia.

PROCURE HACER EERCICIO FISICO AERÓBICO (caminar, carrera suave, ciclismo, natación) a intensidad moderada (65-70 por ciento de frecuencia cardiaca máxima) de tres a cinco veces por semana. Este hábito aumenta el HDL (colesterol bueno) y reduce el LDL (colesterol malo) y los niveles de triglicéridos. 


\section{Referencias bibliográficas}

1. Cómo debe ser la farmacia que necesita la sociedad. Conclusiones de proyecto REFCOM [Internet]. Madrid: Sociedad Española de Farmacia Familiar y Comunitaria; 2014. [Consultado el 21/7/2017]. Disponible en: https:// www.sefac.org/sites/default/files/ sefac2010/private/documentos_sefac/documentos/C\%C3\%B3mo\%20 deber\%20ser $\% 201 \mathrm{a} \% 20$ farmacia $\% 20$ que\%20necesita \%20la\%20sociedad_Conclusiones $\% 20$ proyecto $\% 20$ Refcom.pdf

2. 15 Retos para el presente y futuro de la farmacia Comunitaria [Internet]. Madrid: Sociedad Española de Farmacia Familiar y Comunitaria; 2016. [Consultado el 21/7/2017]. Disponible en: https://www.sefac.org/sites/ default/files/sefac2010/private/documentos_sefac/documentos/15retos. pdf

3. Baixauli VJ, Satué-de-Velasco E, Gil MI, Roig JC, Villasuso B, Sáenz-de-Buruaga S. Propuesta de la Sociedad Española de Farmacia Comunitaria (SEFAC) sobre servicios profesionales farmacéuticos en farmacia comunitaria. Farmacéuticos Comunitarios. 2013 Sep 30; 5(3):119126.

4. Foro de Atención Farmacéutica en Farmacia Comunitaria. Servicios profesionales farmacéuticos asistenciales. Sexto comunicado Foro AF-FC. Farmacéuticos 2016; 419:53-56.

5. Baixauli VJ [Internet]. ¿Qué queremos con los servicios profesionales farmacéuticos? Tribuna SEFAC. [Consultado el 29/7/2017]. Disponible en: https:// www.sefac.org/tribuna-sefac/tribuna-sefac-que-queremos-con-los-servicios-profesionales

6. Gómez JC, Mendoza A, Caelles N, Murillo MD. Programa CESAR. Capa- citación para la prestación del servicio de cesación tabáquica en farmacia comunitaria. Farmacéuticos Comunitarios. 2016 May 26; 8(Supl.1)

7. Gómez Martínez JC [Coordinador]. Documento de Intervención en Cesación Tabáquica en la Farmacia Comunitaria. Madrid: Sociedad Española de Farmacia Familiar y Comunitaria; 2014. ISBN: 978-84-616-98745.

8. Comisión de Servicios Profesionales Farmacéuticos de SEFAC. Especificaciones del servicio de medición y control de la presión arterial mediante MAFC, AMPA y MAPA. Farmacéuticos Comunitarios. 2016 Jun 30; 8(2):16-23. doi:10.5672/FC.21739218.(2016/Vol8).002.03

9. Molinero A, Gómez JC, Iracheta M, Martínez SR, Martell N. Programa impacHta: formación SEFAC $y$ SEH-LELHA en hipertensión y riesgo vascular y capacitación SEFAC para la prestación de servicios de hipertensión y riesgo vascular a farmacéuticos comunitarios. Farmacéuticos Comunitarios. 2016 May 26; 8(Supl.1)

10. Baixauli VJ, Bellver S, Rua FJ, Velasco J, Gómez E, García-Agua N, et al. Proyecto revisa ${ }^{\odot}$. Implantación del servicio RUM en la farmacia comunitaria española. Farmacéuticos Comunitarios. 2016 May 26; 8(Supl.1).

11. Gómez E, Bellver S, Rua FJ, Velasco J, García-Agua N, Romero J, et al. Proyecto REVISA ${ }^{\odot}$. Estudio del impacto del servicio de revisión del uso de los medicamentos en farmacias comunitarias españolas. Farmacéuticos Comunitarios. 2016 May 26; 8(Supl.1).

12. Bellver S, Rua FJ, Velasco J, Gómez E, García-Agua N, Romero J, et al. Programa de capacitación del servicio de revisión del uso de los medicamentos (RUM). Una metodología de aprendizaje novedosa. Farmacéuticos Comunitarios. 2016 May 26; 8(Supl.1).
13. Fornos-Pérez JA, Andrés-Rodríguez NF, Lorenzo-Veiga B, Huarte-Royo J, Vivar-Fernández M, Andrés-Iglesias JC, et al. Detección de personas en riesgo de padecer diabetes en farmacias comunitarias españolas. Farmacéuticos Comunitarios. 2015 Jun 30; 7(2):14-24. doi:10.5672/FC.21739218.(2015/Vol7).002.03

14. Mata M, Artola S, Escalada J, Ezkurra P, Ferrer JC, Fornos JA, et al. Consenso sobre la detección y el manejo de la prediabetes. Grupo de trabajo de consensos y guías clínicas de la Sociedad Española de Diabetes. Farmacéuticos Comunitarios. 2014 Dec 30; 6(4):26-39. doi:10.5672/FC.21739218.(2014/Vol6).004.05

15. Baixauli V, Vanrell B, Llull R, González A, Servera M. Detección y seguimiento de desnutrición en ancianos no institucionalizados por el farmacéutico comunitario en las Islas Baleares. Farmacéuticos Comunitarios. 2017 Mar 30; 9(1):5-13. doi:10.5672/ FC.2173-9218.(2017/Vol9).001.02

16. Moranta FX, Gorreto L, Plaza FJ. Estudio para la detección precoz de EPOC desde las farmacias comunitarias de Illes Balears. FARBALEPOC. Farmacéuticos Comunitarios 2014; 6(Supl.1).

17. Ministerio de Sanidad Servicios Sociales e Igualdad [Internet]. Encuesta Nacional de Salud 2011-2012. 2013. [Consultado 7/7/2017]. Disponible en: http://www.msssi.gob.es/estadEstudios/estadisticas/encuestaNacional/ encuesta2011.htm

18. Castillo D, Burgos F, Guayta R, Giner J, Lozano P, Estrada M, et al. FARMAEPOC group. Airflow obstruction case finding in community pharmacies: A novel strategy to reduce COPD underdiagnosis. Respir Med 2015;109:475-482. doi:10.1016/j. rmed.2015.02.009 